injectable drugs', recommends that 'care must be taken when administering this antiemetic associated with other drugs that prolong the QT interval, namely several cytotoxic agents'. To effectively implement this recommendation, it was thought advisable to point out, in the computerised hospital drug database, all cytotoxic drugs that prolong the OT interval.

Purpose To review all cytotoxic drugs available in the Portuguese pharmaceutical market to identify those with the potential to prolong the OT interval, in order to allow hospital pharmacists to quickly and efficiently implement the above-mentioned recommendation.

Materials and Methods Literature review based upon all summaries of product characteristics (SPCs) of cytotoxic drugs available in Portugal and 48 literature sources from PubMed, found by intersecting the terms 'cytotoxic-induced prolongation of the OT interval', 'antineoplastic-induced prolongation of the QT interval' and 'druginduced prolongation of the OT interval' and using the time limit interval from January/2003 to September/2012.

Results A total of 58 cytotoxic agents currently available in Portugal were investigated. Agents with the potential to prolong the OT interval are: arsenic trioxide, capecitabine, dasatinib, doxorubicin, epirubicin, eribulin, gefitinib, lapatinib, nilotinib, sorafenib, sunitinib and vandetanib. Substantial evidence supports the conclusion that arsenic trioxide and vandetanib have a risk of torsades de pointes (TdP) when used as directed in SPC. Regarding eribulin, lapatinib, nilotinib and sunitinib, there is insufficient evidence that they may cause TdP when used as directed in the SPC. Note that the hormone antagonists bicalutamide and tamoxifen also have the potential to prolong the OT interval.

Conclusions The database produced is a valuable tool to Portuguese hospital pharmacists who dispense cytotoxic drugs, contributing to the implementation of one of the recommendations of the above-mentioned regulation.

No conflict of interest.

\section{GRP-049 DESIGN AND DEVELOPMENT OF A PRESCRIPTION MODULE OF ENTERAL DIETS FOR A NEONATAL UNIT}

doi:10.1136/ejhpharm-2013-000276.049

JJ Martínez Garde, LR López Giménez, M Valero Domínguez, C Abraira Meriel, A Gómez Esteban, MA Martín Vega, MD Rivas Santayana. Hospital Universitario Marqués de Valdecilla, Pharmacy, Santander, Spain

Background A safety problem occurred in requesting enteral diets (EDs) in the neonatal unit. So we decided to develop a special prescription module for requesting EDs.

Purpose To describe the design and development of a prescription and request module for EDs in a neonatal unit.

Materials and Methods The first step was to assemble all the EDs, such as milks, supplements or fortifiers and described the composition of these products, indicating total kilocalories, macronutrients (grammes of protein, lipids and carbohydrates), micronutrients ( $\mathrm{mg}$ and $\mathrm{mEq}$ of $\mathrm{Na}, \mathrm{K}, \mathrm{Cl}, \mathrm{Ca}$ and $\mathrm{Mg}, \mathrm{mg}$ and $\mathrm{mMol}$ of $\mathrm{P}, \mathrm{mg}$ of elemental iron, IU of vitamin D3) and osmolarity ( $\mathrm{mOsm} / \mathrm{L}$ ).

Many of these data weren't in the product's package leaflet, so it was necessary to contact the manufacturer to request this information.

We decided to include the name of the diet, frequency, administration route and type and unit of administration in the ED prescription module.

Results The neonatal computer physician order entry (CPOE) now has another option, the ED module. The prescriptions also include the weight of the patient. When the physicians select ED, they can view the qualitative and quantitative composition of the formula. The prescription module calculates macronutrients provided for that prescription ( $\mathrm{g} / \mathrm{kg} /$ day), micronutrients $(\mathrm{mg} / \mathrm{kg} / \mathrm{day}$,
$\mathrm{mEq} / \mathrm{kg} /$ day or $\mathrm{mMol} / \mathrm{kg} /$ day), total kilocalories ( $\mathrm{kcal} / \mathrm{kg} /$ day) and osmolarity ( $\mathrm{mOsm} / \mathrm{L})$.

The prescribed diet is checked against nutritional requirements obtained from the European Society of Paediatric Gastroenterology and Nutrition guidelines.

Finally, the software can generate the request for the diets without the necessity of handwritten requests.

Conclusions ED can cause medication errors, such as transcription problems, excessive or miscalculated macro and micronutrients or errors in route of administration. These errors may have clinical impact on children and can be more serious in preterm infants. The ED prescription module is an excellent tool to prevent errors and facilitate the nutritional calculations.

No conflict of interest.

\section{GRP-050 DETECTION AND ANALYSIS OF ADVERSE DRUG REACTIONS IN CANCER PATIENTS IN A TERTIARY HOSPITAL}

doi:10.1136/ejhpharm-2013-000276.050

'M Ferrit, 'M Cañadas, 'N Martinez, 'A Madrid, 'E Puerta, 'MS Caparros, 'I Vallejo, ${ }^{2 P}$ Aznarte, ${ }^{2 M}$ Salazar, ${ }^{2} \mathrm{MA}$ Calleja. 'University Hospital Virgen de las Nieves, Pharmacy, Granada, Spain; 'University Hospital Virgen de las Nieves Virgen de las Nieves, Pharmacy, Granada, Spain

Background Adverse drug reactions (ADRs) are especially important with antineoplastic drugs because of their implications on patients' health and quality of life.

Purpose To study the epidemiology, clinical features, diagnosis and pharmacology of ADRs detected in hospitalised patients treated with antineoplastic drugs.

Materials and Methods Analytical observational study (2011). We included all patients receiving cancer treatment. Study variables were: sociodemographic characteristics (age, sex), clinical (diagnostic, stage) and ADRs. The analysis was epidemiological: ADRs conducted (cumulative incidence, CI), clinical: (physiological system affected, type, duration, production mechanism, frequency, severity), pharmacological: (drug, administration, cycle) and diagnostic: (causality, chronological sequence).

Results 125 patients (mean age 51 years), 68\% male, 32\% female, $90 \%$ comorbidities. The most common diagnoses were lymphoma (28\%), specifically non-Hodgkin's Lymphoma (11\%), acute lymphoblastic leukaemia (9\%), acute myeloid leukaemia (6\%) mainly in advanced stages (68\%). We detected a total of $170 \mathrm{ADRs}$ with antineoplastic agents $(28 \% \mathrm{CI})$. Physiological systems primarily affected were: blood (89\%), digestive system (23\%). The most common ADR was cytopenia (49\%) specifically febrile neutropenia (37\%). The duration was $<7$ days $(75 \%)$ and $>7$ days $(25 \%)$. ADRs were mostly produced in a dose-dependent way $(85 \%)$, were very common (94\%) and according to severity were: lethal (2\%), severe $(5 \%)$, moderate $(73 \%)$, mild (19\%). The drugs involved were: cytarabine, methotrexate, idarubicin, carmustine, cisplatin by intravenous administration (97\%) and during first treatment cycles: cycle 1 (53\%), cycle 2 (23\%). $92 \%$ of the ADRs are tested and produced after drug administration (99\%). In $60 \%$ and $19 \%$ of cases the measure was the continuation and discontinuation of antineoplastic therapy, respectively. In cases of re-exposure, the emergence of drug ADRs was positive in $45 \%$ of patients and in the disappearance of ADRs discontinuation was positive in $92 \%$.

Conclusions The incidence of $\mathrm{ADR}$ was high, the majority of ADRs were well known, moderate and positive outcome according to the measurements. It would be better to understand the ADRs as it can help develop other strategies to reduce their impact on the safety of cancer treatments in the first cycles.

No conflict of interest. 